\title{
ON THE CONNECTEDNESS OF THE SET OF RIEMANN SURFACES WITH REAL MODULI
}

\author{
ANTONIO F. COSTA AND RUBÉN A. HIDALGO
}

\begin{abstract}
The moduli space $\mathcal{M}_{g}$, of genus $g \geq 2$ closed Riemann surfaces, is a complex orbifold of dimension $3(g-1)$ which carries a natural real structure i.e. it admits an anti-holomorphic involution $\sigma$. The involution $\sigma$ maps each point corresponding to a Riemann surface $S$ to its complex conjugate $\bar{S}$. The fixed point set of $\sigma$ consists of the isomorphism classes of closed Riemann surfaces admitting an anticonformal automorphism. Inside $\operatorname{Fix}(\sigma)$ is the locus $\mathcal{M}_{g}(\mathbb{R})$, the set of real Riemann surfaces, which is known to be connected by results due to P. Buser, M. Seppälä and R. Silhol. The complement $\operatorname{Fix}(\sigma)-\mathcal{M}_{g}(\mathbb{R})$ consists of the so called pseudo-real Riemann surfaces, which is known to be non-connected. In this short note we provide a simple argument to observe that $\operatorname{Fix}(\sigma)$ is connected.
\end{abstract}

\section{INTRODUCTION}

The moduli space $\mathcal{M}_{g}$, of genus $g \geq 2$ closed Riemann surfaces, is a complex orbifold of dimension $3(g-1)$. The study of this moduli space was already started by F. Klein. This space carries a natural real structure given by an involution $\sigma$ which sends each Riemann surface to its complex conjugate. The fixed point set of $\sigma$ consists of the isomorphism classes of closed Riemann surfaces admitting an anticonformal automorphism and those surfaces are said to have real moduli. The quotient space $\mathcal{M}_{g} /\langle\sigma\rangle$ is the moduli space of Riemann surfaces of genus $g$ considered as Klein surfaces, i. e. two surfaces are equivalent if they are holomorphic or anti-holomorphically equivalent. Inside $\operatorname{Fix}(\sigma)$ is the locus $\mathcal{M}_{g}(\mathbb{R})$, consisting of those admitting an anticonformal involution (Riemann surfaces corresponding to real algebraic curves), which is known to be connected by results due to P. Buser, M. Seppälä and R. Silhol $[4,8]$ (see also a proof in [5]). The locus $\mathcal{M}_{g}(\mathbb{R})$ has been studied by Klein in [7] and the fact that in general $\operatorname{Fix}(\sigma) \neq \mathcal{M}_{g}(\mathbb{R})$ was noted by C. Earle in [6]. The complement $\mathcal{P}_{g}=\operatorname{Fix}(\sigma)-\mathcal{M}_{g}(\mathbb{R})$ consists of the so called pseudo-real Riemann surfaces. In [1] it was observed that $\mathcal{P}_{g}$ is non-empty for every $g \geq 2$ (then $\operatorname{Fix}(\sigma) \neq \mathcal{M}_{g}(\mathbb{R})$ for all $g \geq 2$ ). In [2] it was observed that $\mathcal{P}_{2}$ and $\mathcal{P}_{3}$ are connected and $\mathcal{P}_{4}$ is non-connected (it has three connected components). There are infinite many integers $n_{i}$ such that $\mathcal{P}_{n_{i}}$ is not connected (this fact follows from Theorems 3.4, 5.4, 5.5 and Section 6 of [1] to construct families of

Partially supported by Project MTM2014-55812-P (Spanish Ministry of Competitivity), Project of Fondecyt 1150003 and Project Anillo ACT1415 PIA CONICYT. 
pseudo-real surfaces and from the Corollary of Theorem 2 of [9] to prove that these families are in different connected components).

In this short note we provide a simple argument to observe that $\operatorname{Fix}(\sigma)$ is connected.

Theorem 1. The set $\operatorname{Fix}(\sigma)$ is connected.

This connectedness fact may not be a surprise, but it seems it has not been noted in the existent literature.

Acknowledgements. We wish to thank the referee for corrections and suggestions.

\section{The SET of Riemann SURfaCes With REAL MOdUli IS CONNECTED}

In this section, we proceed to prove Theorem 1. Let $S$ be a closed Riemann surface of genus $g \geq 2$, admitting an anticonformal automorphism $\tau$ of order $2 n$, where $n \geq 2$ is even. The quotient orbifold $\mathcal{O}=S /\langle\tau\rangle$ is homeomorphic to the connected sum of some $\gamma$ real projective planes and has exactly $r$ cone points, say of orders $n_{1}, \ldots, n_{r} \in$ $\{2, \ldots, n\}$, where each $n_{j}$ is a divisor of $n$. This means that there is an NEC group $\Delta$, acting on the unit disc $\mathbb{D}$, with signature $\left(\gamma ;-;\left[n_{1}, \ldots, n_{r}\right]\right)$ and there is a surjective homomorphism $\rho: \Delta \rightarrow C_{2 n}=\langle\tau\rangle$, whose kernel $\Gamma$ is a Fuchsian group uniformizing $S$ and $\langle\tau\rangle$ is induced by $\Delta$, that is, $\mathbb{D} / \Gamma=S \rightarrow \mathbb{D} / \Delta=S /\langle\tau\rangle$.

The locus $\mathcal{O}(S, \tau)$ in moduli space $\mathcal{M}_{g}$ consisting of those (classes of) closed Riemann surfaces $\widehat{S}$ admitting an anticonformal automorphism $\widehat{\tau}$ of order $2 n$ so there is an orientation preserving homeomorphism $\phi: S \rightarrow \widehat{S}$ conjugating $\tau$ to $\widehat{\tau}$ is connected [2]. Now, since the locus $\mathcal{M}_{g}(\mathbb{R})$ is connected, in order to check the connectivity of Fix $(\sigma)$, we only need to find a point $[\widehat{S}] \in \mathcal{O}(S, \tau)$ so that $\widehat{S}$ admits also an anticonformal involution. In the NEC group setting, this is equivalent to finding an NEC group $K$ containing reflections (i.e. the group $K$ uniformizes a bordered Klein surface) and a subgroup $\widehat{\Delta}$ of $K$ so that there is an isomorphism $\iota: \Delta \rightarrow \widehat{\Delta}$ with $\iota(\Gamma)$ being a normal subgroup of $K$. Note that $\iota(\Gamma)$ is a Fuchsian group with $\widehat{S}=\mathbb{D} / \iota(\Gamma)$ a closed Riemann surface which has an automorphism $\widehat{\tau}$ topologically equivalent to $\tau$ $(\widehat{\tau}$ is an automorphism group of the cyclic covering $\mathbb{D} / \iota(\Gamma)=\widehat{S} \rightarrow \mathbb{D} / \widehat{\Delta})$ and $\widehat{S}$ has anticonformal involutions too, produced by the lifting of the reflections of $K$ to $\widehat{S}$ (note that $\widehat{S}$ has empty boundary but $\mathbb{D} / K$ is bordered). In this way we have $\mathcal{O}(S, \tau) \cap \mathcal{M}_{g}(\mathbb{R}) \neq \varnothing$ for every pseudo-real surface $S$; this implies $\operatorname{Fix}(\sigma)$ is connected.

2.1. The construction of $K$. Let $K$ be an NEC group uniformizing the closed disc, with $\gamma$ interior cone points of order 2 and $r$ cone points in its border of orders $n_{1}, \ldots, n_{r}$, that is, an NEC group of signature $\left(0 ;+;[2, \gamma, 2],\left\{\left(n_{1}, \ldots, n_{r}\right)\right\}\right)$. A canonical presentation for $K$ is as follows

$$
\begin{gathered}
K=\left\langle x_{1}, \ldots, x_{\gamma}, e, \tau_{1}, \ldots, \tau_{r+1}: x_{1}^{2}=\cdots=x_{\gamma}^{2}=\tau_{1}^{2}=\cdots=\tau_{r+1}^{2}=1,\right. \\
\left.e^{-1} \tau_{r+1} e=\tau_{1}, x_{\gamma} \cdots x_{2} x_{1} e=1,\left(\tau_{1} \tau_{2}\right)^{n_{1}}=\cdots=\left(\tau_{r} \tau_{r+1}\right)^{n_{r}}=1\right\rangle,
\end{gathered}
$$




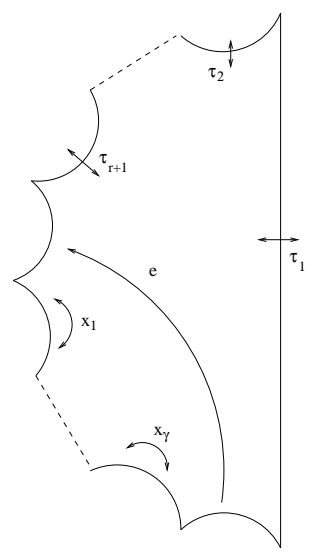

Figure 1. A fundamental domain for $K$

where the elements $x_{j}$ are elliptic transformations of order two, the elements $\tau_{j}$ are reflections and $e$ is an hyperbolic or elliptic element (see [3] and Figure 1 for a fundamental domain of $K$ ).

2.2. A subgroup $\widehat{\Delta}$ of $K$. Let us consider the surjective homomorphism

$$
\begin{gathered}
\theta: K \rightarrow C_{2}=\left\langle a: a^{2}=1\right\rangle \\
x_{1}, \ldots, x_{\gamma}, \tau_{1}, \ldots, \tau_{r+1} \mapsto a, e \mapsto 1 .
\end{gathered}
$$

The kernel $\widehat{\Delta}$ of $\theta$ has no reflections and contains orientation reversing elements (for instance $\left.c_{1} x_{1}\right)$; so its signature must be of the form $\left(h ;-;\left[m_{1}, \ldots, m_{s}\right]\right)$, that is, $\mathbb{D} / \widehat{\Delta}$ is the connected sum of $h$ real projective planes and contains exactly $s$ cone points, these having orders $m_{1}, \ldots, m_{s}$. Using the Riemann-Hurwitz formula and the usual methods to compute the signature of an NEC subgroup (see [3]), we have that $h=\gamma, s=r$ and $n_{j}=m_{j}$. So, $\Delta$ is isomorphic to $\widehat{\Delta}$; let $\iota: \Delta \rightarrow \widehat{\Delta}$ be such an isomorphism. A fundamental domain for $\widehat{\Delta}$ is shown in Figure 2, this given as the union of the previous fundamental domain for $K$ with its image under the reflection $\tau_{1}$. By the Poincaré polygon theorem (or using the Schreier-Reidemeister method) a presentation of $\widehat{\Delta}$, in terms of the generators of $K$, may be obtained. We have as generators

$$
\delta_{1}=\tau_{1} x_{1}, \ldots, \delta_{\gamma}=\tau_{1} x_{\gamma}, c_{1}=\tau_{1} \tau_{2}, \ldots, c_{r}=\tau_{1} \tau_{r+1}, e_{1}=e, e_{2}=\tau_{1} e \tau_{1}
$$

satisfying the following relations

$$
\left.\left.\begin{array}{c}
c_{1}^{n_{1}}=1 \\
\left(c_{1}^{-1} c_{2}\right)^{n_{2}}=\left(c_{2}^{-1} c_{3}\right)^{n_{3}} \cdots=\left(c_{r-1}^{-1} c_{r}\right)^{n_{r}}=1, \\
e_{1} e_{2}^{-1} c_{r}=1 \\
\delta_{1}^{-1} \delta_{2} \delta_{3}^{-1} \cdots \delta_{\gamma-1}^{-1} \delta_{\gamma}=e_{1} \\
\delta_{1} \delta_{2}^{-1} \delta_{3} \cdots \delta_{\gamma-1} \delta_{\gamma}^{-1}=e_{2}
\end{array}\right\} \text { (if } \gamma \text { is even }\right)
$$




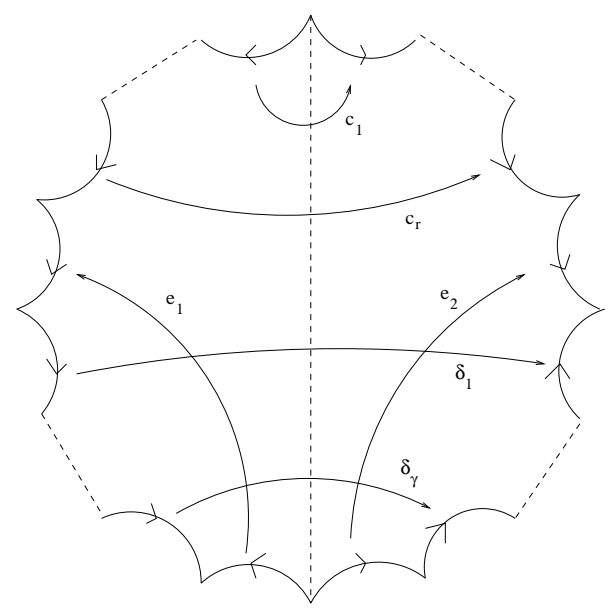

Figure 2. A fundamental domain for $\widehat{\Delta}$

$$
\left.\left.\begin{array}{ll}
\delta_{1} \delta_{2}^{-1} \delta_{3} \cdots \delta_{\gamma-1}^{-1} \delta_{\gamma} & =e_{1} \\
\delta_{1}^{-1} \delta_{2} \delta_{3}^{-1} \cdots \delta_{\gamma-1} \delta_{\gamma}^{-1} & =e_{2}
\end{array}\right\} \text { (if } \gamma \text { is odd }\right)
$$

2.3. The final step. Let us consider the surjective homomorphism $\eta=\rho \circ \iota^{-1}: \widehat{\Delta} \rightarrow$ $C_{2 n}$, whose kernel is $\widehat{\Gamma}=\iota(\Gamma)$; a torsion free Fuchsian group that uniformizes a closed Riemann surface $\widehat{S}$. In order to finish our proof, we only need to check that $\widehat{\Gamma}$ is a normal subgroup of $K$. This is what the following general lemma asserts.

Lemma 1. Let $A$ be an abelian group and let $\zeta: \widehat{\Delta} \rightarrow A$ be a homomorphism. Then $e_{1} e_{2} \in \operatorname{ker}(\zeta)$ and $\operatorname{ker}(\zeta) \triangleleft K$.

Proof. We assume $\gamma$ even; the odd case is similar. The relations

$$
\delta_{1}^{-1} \delta_{2} \delta_{3}^{-1} \cdots \delta_{\gamma-1}^{-1} \delta_{\gamma}=e_{1}, \text { and } \delta_{1} \delta_{2}^{-1} \delta_{3} \cdots \delta_{\gamma-1} \delta_{\gamma}^{-1}=e_{2},
$$

assert that $\zeta\left(e_{1}\right)=\zeta\left(e_{2}\right)^{-1}$, so, $\zeta\left(e_{1} e_{2}\right)=1$. Next, since

$$
\begin{gathered}
\tau_{1} \delta_{j} \tau_{1}=\delta_{j}^{-1}, j=1, \ldots, \gamma, \\
\tau_{1} c_{k} \tau_{1}=c_{k}^{-1}, k=1, \ldots, r, \\
\zeta\left(e_{1}\right)=\zeta\left(e_{2}\right)^{-1}, \\
\widehat{\Delta}^{\prime} \triangleleft \operatorname{ker}(\zeta) \text { (as } A \text { is an abelian group), }
\end{gathered}
$$

we may see that $\tau_{1}$ induces the inverse automorphism of $A$, i.e.,

$$
a \in A \mapsto a^{-1} \in A
$$

In particular, $\tau_{1} \operatorname{ker}(\zeta) \tau_{1}=\operatorname{ker}(\zeta)$. Since, $K=\left\langle\widehat{\Delta}, \tau_{1}\right\rangle$, we obtain that $\operatorname{ker}(\zeta) \triangleleft K$.

Corollary 1. If $S$ is a pseudo-real Riemann surface admitting an anticonformal automorphism $\tau$ of order $2 n$, then there is a real Riemann surface $\widehat{S}$ admitting an anticonformal automorphism $\widehat{\tau}$ such that $(\widehat{S}, \widehat{\tau})$ is topologically conjugate to $(S, \tau)$. 
Proof. The surface $\widehat{S}$ is uniformized by $\operatorname{ker}(\eta)$ and we use $\eta=\zeta, A=C_{2 n}$, in the above lemma.

Note that if $\widehat{S}$ is the surface given in the above Corollary $D_{2 n} \leq \operatorname{Aut}(\widehat{S})$.

For every pseudo-real Riemann surface $S$, Corollary $1 \operatorname{implies} \mathcal{O}(S, \tau) \cap \mathcal{M}_{g}(\mathbb{R}) \neq \varnothing$ and that $\operatorname{Fix}(\sigma)$ is connected.

\section{REFERENCES}

[1] E. Bujalance, M. Conder and A. F. Costa. Pseudo real Riemann surfaces and chiral regular maps. Trans. Amer. Math. Soc. 362 No. 7 (2010), 3365-3376.

[2] E. Bujalance and A. F. Costa. Automorphism groups of pseudo-real Riemann surfaces of low genus. Acta Math. Sin. (English Series) 30 No. 1 (2014), 11-22.

[3] E. Bujalance, J. J. Etayo, J. M. Gamboa, G. Gromadzki, Automorphism groups of compact bordered Klein surfaces. A combinatorial approach. Lecture Notes in Mathematics, 1439. SpringerVerlag, Berlin, 1990. xiv+201 pp. ISBN: 3-540-52941-1

[4] P. Buser, M. Seppälä and R. Silhol. Triangulations and moduli space of Riemann surfaces with group actions. Manuscripta Math. 88 (1995), 209-224.

[5] A. F. Costa and M. Izquierdo On the connectedness of the locus of real Riemann surfaces. Ann. Acad. Sci. Fenn. Math. 27 (2002), no. 2, 341-356.

[6] C. J. Earle, On moduli of closed Riemann surfaces with symmetries. In "Advances in the Theory of Riemann surfaces", Annals of Mathematics Studies 66, pages 119-130, Princeton University Press and University of Tokyo Press, Princeton New Yersey, 1971.

[7] F. Klein, Über Realitätverhältnisse bei der einem beliebigen Geschlechte zugehörigen Normalkurve der $\varphi$. Math. Ann. 42 (1892), 1-29.

[8] M. Seppälä. Real algebraic curves in the moduli space of complex curves. Comp. Math. 74 (1990), 259-283.

[9] D. Singerman. Symmetries and pseudosymmetries of hyperelliptic surfaces. Glasgow Math. J. 21 (1980), no. 1, 39-49.

Departamento de Matemáticas Fundamentales, Facultad de Ciencias, Uned, 28040 MADRID, SPAIN

E-mail address: acosta@mat.uned.es

Departamento de Matemática y Estadística, Universidad de la Frontera. Temuco, CHILE

E-mail address: ruben.hidalgo@ufrontera.cl 